\title{
Quasi-Periodicity and Border-Collision Bifurcations in a DC-DC Converter with Pulsewidth Modulation
}

Zhusubalaliyev, Zh. T.; Soukhoterin, E.A.; Mosekilde, Erik

Published in:

I E E E Transactions on Circuits and Systems Part 1: Regular Papers

Link to article, DOI:

10.1109/TCSI.2003.815196

Publication date:

2003

Document Version

Publisher's PDF, also known as Version of record

Link back to DTU Orbit

Citation (APA):

Zhusubalaliyev, Z. T., Soukhoterin, E. A., \& Mosekilde, E. (2003). Quasi-Periodicity and Border-Collision Bifurcations in a DC-DC Converter with Pulsewidth Modulation. I E E E Transactions on Circuits and Systems Part 1: Regular Papers, 50(8), 1047-1057. https://doi.org/10.1109/TCSI.2003.815196

\section{General rights}

Copyright and moral rights for the publications made accessible in the public portal are retained by the authors and/or other copyright owners and it is a condition of accessing publications that users recognise and abide by the legal requirements associated with these rights.

- Users may download and print one copy of any publication from the public portal for the purpose of private study or research.

- You may not further distribute the material or use it for any profit-making activity or commercial gain

- You may freely distribute the URL identifying the publication in the public portal 


\title{
Quasi-Periodicity and Border-Collision Bifurcations in a DC-DC Converter With Pulsewidth Modulation
}

\author{
Zhanybai T. Zhusubaliyev, Evgeniy A. Soukhoterin, and Erik Mosekilde
}

\begin{abstract}
The paper considers the dynamics of a dc-dc converter with pulsewidth modulation. The typical scenario for the transition to chaos in such systems proceeds via quasi-periodicity, resonance cycles, and torus destruction. Detailed bifurcation analysis shows that the resonance solutions arise via border-collision bifurcations (BCB) on a two-dimensional torus. The arrangement of the resonance domains within the parameter plane is related to the Farey series, and their internal structure is described. It is shown that transitions to chaos mainly occur through finite sequences of BCB. Some other possible causes of complex dynamics are considered, including a subcritical Neimark-Sacker (Andronov-Hopf) bifurcation and the associated hysteretic phenomena.
\end{abstract}

Index Terms-Border-collision bifurcations (BCB), chaos, dc-dc converters, piecewise-smooth systems, power electronics, quasi-periodicity.

\section{INTRODUCTION}

$\mathbf{M}$ ANY problems in engineering and applied science result in the consideration of piecewise-smooth dynamical systems. Examples are relay and pulsewidth-modulated control systems, impact oscillators, power converters, and various electronic circuits with piecewise-smooth characteristics.

Transitions from periodic to chaotic oscillations in piecewisesmooth dynamical systems can display a variety of peculiarities connected with a specific type of bifurcations that cannot occur in smooth dynamical systems. These are the so-called bordercollision bifurcations (BCB) [1]-[8]. Previous studies by Feigin [1]-[4], Nusse and Yorke [5], [6], Nusse et al. [7], Banerjee et al. [9]-[12], di Bernardo et al. [8], [13], [14], and Zhusubaliyev et al. [15]-[17], show that BCB cover an extremely broad variety of bifurcation phenomena and transitions to chaos. A complete classification of these transitions has not yet been performed. However, stimulated by possible applications in many fields of engineering and applied science, the investigation of $\mathrm{BCB}$ in piecewise-smooth differential systems and piecewise-differentiable maps has recently attracted significant interest (see, e.g., [18]-[20]).

Since first reported by Hamill [21], bifurcation phenomena and chaotic oscillations in dc-dc converters with pulsewidth modulation (PWM) have been studied in numerous works

Manuscript received August 12, 2002; revised April 11, 2003. This paper was recommended by Guest Editor M. di Bernardo.

Z. Zhusubaliyev and E. Soukhoterin are with the Department of Computer Science, Kursk State Technical University, 305040 Kursk, Russia (e-mail: cct@cafct.kursk.ru).

E. Mosekilde is with the Center for Chaos and Turbulence Studies, Department of Physics, Technical University of Denmark, 2800 Lyngby, Denmark, (e-mail: Erik.Mosekilde@fysik.dtu.dk).

Digital Object Identifier 10.1109/TCSI.2003.815196
[9]-[12], [15]-[20], [22], and [23]. di Bernardo and Tse [20] provide a collection of main results in the fields of numerical and experimental investigation of chaotic behavior in $\mathrm{dc}-\mathrm{dc}$ converters (see also [24]). Among the earliest works providing a detailed numerical and analytical investigation of bifurcations and transitions to chaos in dc-dc converters we should recall the paper by Baushev and Zhusubaliyev [25] (see also [26]). These authors showed that such systems can display a situation where there is a wide range of parameters with many locally stable limit cycles with different dynamic characteristics, including regions of coexistence. These cycles arise in hard transitions, for example, through a saddle node or BCB, and with changing parameters each of them can undergo either a finite or an infinite sequence of period-doubling bifurcations, resulting in the transition to chaos. Similar results were obtained by Fossas and Olivar [27] using an approach similar to the one described in the work by Baushev and Zhusubaliyev [25]. Complex behavior of switching power converters connecting with $\mathrm{BCB}$ and grazing bifurcations were described by numerous researchers [9]-[12], [15]-[20].

El Aroudi and Leyva [23] studied quasi-periodic behavior in a pulsewidth modulated dc-dc boost converter. The observed phenomena include a subcritical torus birth (Neimark-Sacker or Andronov-Hopf) bifurcation, the associated hysteretic transitions, and the onset of chaos via torus destruction. The authors described two different ways for the destruction of the torus: local period doubling of resonance cycles, and loss of the torus smoothness (see also [28]).

In this paper, we present the results of an investigation of a number of new phenomena that can arise in piecewise-smooth dynamical systems, whose motion involves two (or more) periodic components. When two self-oscillatory systems interact (or a self-oscillatory system is subjected to an external periodic forcing), the total motion can be viewed as occurring on the surface of a two-dimensional (2-D) torus and, in the absence of resonances, the motion is said to be quasi-periodic. The problem that we are interested in, is associated with the role that $\mathrm{BCB}$ play in the synchronization of the two modes and in the transition to chaos when the torus finally breaks down.

To examine these phenomena, we shall consider a three-dimensional (3-D) model of a dc-dc buck converter with PWM whose behavior is described by a 3-D piecewise-smooth system of nonautonomous differential equations.

It is well known that resonance phenomena, which always precede the appearance of chaos, play an important role in the quasi-periodic scenario for the transition to chaos via torus destruction. For smooth dynamical systems, the basic theorem for the destruction of a 2-D torus was proved and the possible ways 


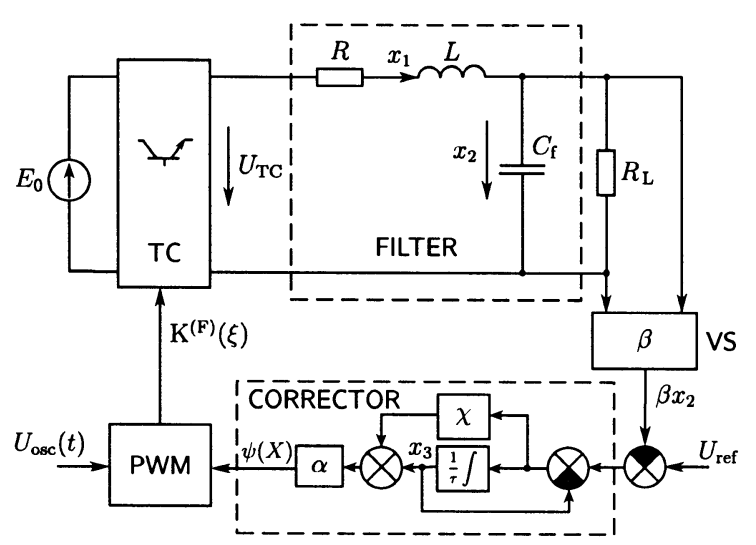

Fig. 1. Coupling diagram for a dc-dc converter.

for the appearance of chaotic dynamics were described in the important work of Afraimovich and Shil'nikov [29].

The generic character of the Afraimovich and Shil'nikov analysis has been confirmed numerically as well as experimentally for a wide class of discrete time and flow systems [30]-[34], clearly demonstrating the possible routes to disintegration for a 2-D torus in smooth systems.

One of the main aims of our investigation is to show that the transitions to chaos through two-frequency quasi-periodicity in piecewise-smooth systems can differ fundamentally from the mechanisms described in the existing literature (see also [16]). At the same time we show that the boundaries of the Arnol'd tongues for nonsmooth systems are formed by BCB, rather than by the saddle-node bifurcations known from smooth systems. Moreover, the internal organization of the tongues is also quite different from the case of smooth systems.

\section{Mathematical Model AND Methods of BIFURCATION ANALYSIS}

Fig. 1 shows the coupling diagram for a dc-dc buck converter with proportional-plus-integrating (PI) feedback regulation. Such converters are well known in power electronics, where they are used to convert a dc voltage into a dc voltage of another value (e.g., buck converters, buck-boost converters, boost converters, etc.). Typical applications are power supplies for industrial and aerospace equipment as well as for domestic electronic appliances and computer equipment [35]-[37].

DC-DC converters are often controlled through some kind of PWM. There is no generally accepted classification of the different types of PWM. However, most specialists in the fields of power engineering and control theory seem to follow the classification suggested by Tsypkin [38] (see also [39], [40]). In this paper, we will also follow this classification.

In Fig. 1, TC is the transistor converter, and PWM is the modulator which operates in accordance with the algorithm for two-sided PWM of the first kind. This implies that the transistor converter is forcedly turned on in the beginning of each clock interval and turns off when the sawtooth signal $U_{\text {osc }}$ exceeds the value of the feedback signal in the beginning of the clock interval. The converter is off as long as the sawtooth voltage remains larger than the feedback signal in the beginning of the

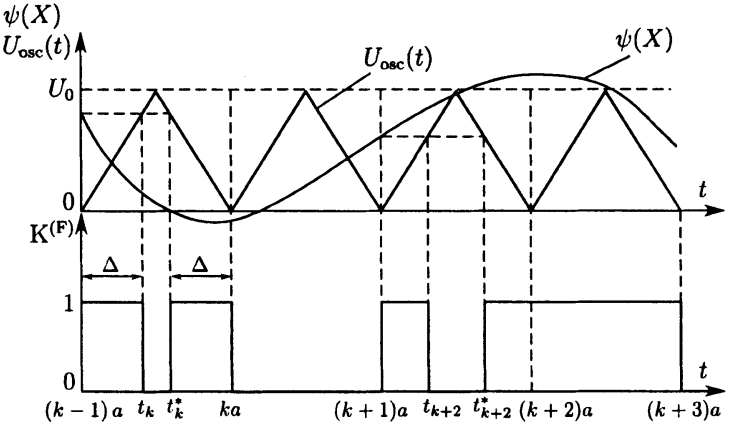

Fig. 2. Generation of control pulses for a dc-dc converter.

clock interval and then turns on. This leads to two symmetrical control pulses during the clock interval [40]. FILTER is a standard low-pass filter, VS is the voltage sensor, and CORRECTOR represents a PI-feedback link. $\beta$ is the transfer constant of the voltage sensor, $\psi(X)=\alpha\left(\chi\left(U_{\text {ref }}-\beta x_{2}\right)+(1-\right.$ $\left.\chi) x_{3}\right)$ is the output signal of the PI corrector, and $U_{\text {osc }}(t)$ is the external periodic action that has the form of a sawtooth voltage, see Fig. 2. $\alpha$ and $\chi$ are the amplification and transfer constants of the corrector, and $\mathrm{K}^{(\mathrm{F})}$ is the output signal of the pulsewidth modulator. During the time interval $[(k-1) a, k a]$, $k=1,2,3, \ldots$, the expression for $U_{\mathrm{osc}}(t)$ takes the form

$U_{\mathrm{osc}}(t)=U_{0} \times\left\{\begin{array}{l}\frac{2 t}{a}-\left[\frac{2 t}{a}\right], \quad(k-1) a \leq t<\left(k-\frac{1}{2}\right) a \\ 1-\left(\frac{2 t}{a}-\left[\frac{2 t}{a}\right]\right), \quad\left(k-\frac{1}{2}\right) a \leq t<k a .\end{array}\right.$

Here, $U_{0}$ is the amplitude of the external periodic action and $a$ is the action period (modulation period). Square brackets mean that one has to take the integer part of the argument. Only the front of the external action $U_{\text {osc }}(t)$ is used to determine the switching instant (see. Fig. 2). Hence, we only need the expression describing the front, i.e., $U_{\mathrm{osc}}(t)=U_{0}(2 t / a-[2 t / a])$.

The equations of motion for the pulsewidth-modulated converter constitute a set of three ordinary differential equations with discontinuous right-hand sides

$$
\frac{\mathrm{d} X}{\mathrm{~d} t}=A X+B(t, X)
$$

with

$$
\begin{aligned}
X & =\left(x_{1}, x_{2}, x_{3}\right)^{\mathrm{T}} \\
A & =\left(\begin{array}{ccc}
-\frac{R}{L} & -\frac{1}{L} & 0 \\
\frac{1}{C_{\mathrm{f}}} & -\frac{1}{C_{\mathrm{f}} R_{\mathrm{L}}} & 0 \\
0 & -\frac{\beta}{\tau} & -\frac{1}{\tau}
\end{array}\right) \\
B(t, X) & =\left(\begin{array}{c}
\frac{E_{0}}{L} \mathrm{~K}^{(\mathrm{F})}(\xi) \\
0 \\
\frac{U_{\mathrm{ref}}}{\tau}
\end{array}\right) .
\end{aligned}
$$

Here, $x_{1}$ and $x_{2}$ are the current in the inductance coil and the voltage on the capacitor of the $L C$ filter, respectively, and $x_{3}$ is the voltage of the corrector's integrator. The superscript $\mathrm{T}$ denotes the operation of transposition. $R_{\mathrm{L}}$ is the load resistance, and $\tau$ is the time constant of the corrector. $E_{0}$ is the input voltage, $\xi(t, X)=\psi(X)-U_{\text {osc }}(t)$, and $U_{\text {ref }}$ is the reference voltage (see Fig. 1). 
Within the clock interval $[(k-1) a, k a], k=1,2,3, \ldots$ the output signal of the pulsewidth modulator $\mathrm{K}^{(\mathrm{F})}(\xi)$ is given by (see Fig. 2)

$$
\mathrm{K}^{(\mathrm{F})}(\xi)= \begin{cases}1, & (k-1) a<t<t_{k} \\ 0, & t_{k}<t<t_{k}^{*} \\ 1, & t_{k}^{*}<t<k a .\end{cases}
$$

The modulator switching instants $t_{k}$ and $t_{k}^{*}$ are determined according to the algorithm of PWM of the first kind [38]-[40]

$$
\begin{aligned}
& t_{k}=\left\{\begin{array}{cc}
(k-1) a, & \xi\left((k-1) a, X_{k-1}\right) \leq 0 \\
(k-1) a+\Delta, & {\left[\xi\left((k-1) a, X_{k-1}\right)>0\right]} \\
\left(k-\frac{1}{2}\right) a, & \vee\left[\xi\left(\left(k-\frac{1}{2}\right) a, X_{k-1}\right)<0\right] \\
\xi\left(t, X_{k-1}\right)>0 \forall t: & (k-1) a<t<\left(k-\frac{1}{2}\right) a
\end{array}\right. \\
& t_{k}^{*}=(2 k-1) a-t_{k} .
\end{aligned}
$$

Here, $\Delta=(1 / 2) a \psi\left(X_{k-1}\right) / U_{0}$ is the pulse duration $(0<\Delta<a / 2)$. In the first case, $\Delta=0$ (see the range $[k a,(k+1) a]$ in Fig. 2) and in the third case $\Delta=a / 2$ (see the range $[(k+2) a,(k+3) a]$ in Fig. 2).

In this analysis, we have assumed the following parameter values. $E_{0}=104 \mathrm{~V}, R=10.6 \Omega, L=0.1 \mathrm{H}, C_{\mathrm{f}}=10^{-6} \mathrm{~F}$, $R_{\mathrm{L}}=100 \Omega, U_{0}=10 \mathrm{~V}, U_{\text {ref }}=5 \mathrm{~V}, \beta=0.1, a=10^{-4} \mathrm{~s}$, and $\tau=4 \cdot 10^{-4} \mathrm{~s}$. These parameter values correspond to the parameters of an actual dc-dc converter [40]. We will consider the amplification and transfer constants of the corrector $\alpha>0$ and $0<\chi<1$ as the control parameters.

With the assumed parameter values, the eigenvalues $\lambda_{1}, \lambda_{2}$, and $\lambda_{3}$ of the matrix $A$ are real and negative

$$
\lambda_{1}=\sigma_{1}+\sigma_{2}, \lambda_{2}=\sigma_{1}-\sigma_{2} \quad \lambda_{3}=\frac{-1}{\tau}
$$

with

$$
\begin{aligned}
\sigma_{1} & =-\frac{1}{2}\left(\frac{R}{L}+\frac{1}{C_{\mathrm{f}} R_{\mathrm{L}}}\right) \\
\sigma_{2} & =-\sqrt{\frac{1}{4}\left(\frac{R}{L}+\frac{1}{C_{\mathrm{f}} R_{\mathrm{L}}}\right)^{2}-\frac{1}{L C_{\mathrm{f}}}\left(1+\frac{R}{R_{\mathrm{L}}}\right)} .
\end{aligned}
$$

Therefore, in order to make the analysis easier, we can replace (1) by the following simpler system [17], [25]:

$$
\left\{\begin{array}{l}
\frac{\mathrm{d} w_{1}}{\mathrm{~d} t}=\lambda_{1}\left(w_{1}-\mathrm{K}^{(\mathrm{F})}(\xi)\right) \\
\frac{\mathrm{d} w_{2}}{\mathrm{~d} t}=\lambda_{2}\left(w_{2}-\mathrm{K}^{(\mathrm{F})}(\xi)\right) \\
\frac{\mathrm{d} w_{3}}{\mathrm{~d} t}=\lambda_{3}\left(w_{3}-\mathrm{K}^{(\mathrm{F})}(\xi)\right)
\end{array}\right.
$$

$\xi\left(t, w_{1}, w_{2}, w_{3}\right)=\sum_{i=1}^{3} \gamma_{i}\left(\chi-\frac{\lambda_{3}}{\lambda_{i}}\right) w_{i}-\frac{Q}{\alpha}\left(\frac{2 t}{a}-\left[\frac{2 t}{a}\right]\right)+q$

with

$$
\begin{aligned}
\gamma_{1} & =\frac{\lambda_{1}}{\lambda_{1}-\lambda_{3}} \\
\gamma_{2} & =\frac{\lambda_{1}}{\lambda_{3}-\lambda_{2}} \\
\gamma_{3} & =-\left(\gamma_{1}+\gamma_{2}\right) \\
Q & =\frac{U_{0}\left(\lambda_{1}-\lambda_{2}\right)\left(R_{\mathrm{L}}+R\right)}{\beta E_{0} \lambda_{2} R_{\mathrm{L}}} \approx 6.48 \\
q & =\frac{U_{\text {ref }}}{U_{0}} Q \approx 3.24 .
\end{aligned}
$$

The variables $\left(w_{1}, w_{2}, w_{3}\right)$ are connected with the original variables $\left(x_{1}, x_{2}, x_{3}\right)$ through the linear transformation

$$
\begin{aligned}
& x_{1}=\frac{\frac{E_{0}}{L}}{\lambda_{1}-\lambda_{2}}\left(\frac{\lambda_{2}+\frac{R}{L}}{\lambda_{1}} w_{1}-\frac{\lambda_{1}+\frac{R}{L}}{\lambda_{2}} w_{2}\right) \\
& x_{2}=\frac{\frac{E_{0}}{(L C)}}{\lambda_{2}-\lambda_{1}}\left(\frac{w_{1}}{\lambda_{1}}-\frac{w_{2}}{\lambda_{2}}\right) \\
& x_{3}=-\lambda_{3} \sum_{i=1}^{3} \mu_{i} \frac{w_{i}}{\lambda_{i}}+U_{\text {ref }}
\end{aligned}
$$

where

$$
\begin{aligned}
\mu_{1} & =-\frac{\beta E_{0}}{L C\left(\lambda_{2}-\lambda_{1}\right)\left(\lambda_{1}-\lambda_{3}\right)} \\
\mu_{2} & =\frac{\beta E_{0}}{L C\left(\lambda_{2}-\lambda_{1}\right)\left(\lambda_{2}-\lambda_{3}\right)} \\
\mu_{3} & =-\left(\mu_{1}+\mu_{2}\right) .
\end{aligned}
$$

By virtue of the periodic forcing, the dynamical system (2) may be reduced to the 3-D stroboscopic mapping

$$
\begin{gathered}
w_{i k}=\mathrm{e}^{a \lambda_{i}} w_{i(k-1)}+\mathrm{e}^{a \lambda_{i}\left(1-z_{k}\right)}-\mathrm{e}^{a \lambda_{i} z_{k}}+1-\mathrm{e}^{a \lambda_{i}} \\
w_{i k}=w_{i}(k a) ; i=1,2,3 ; k=1,2, \ldots \\
z_{k}= \begin{cases}0, & \varphi(0) \leq 0 \\
z^{*}, & (\varphi(0)>0) \vee\left(\varphi\left(\frac{1}{2}\right)<0\right) \\
\frac{1}{2}, & \varphi\left(\frac{1}{2}\right) \geq 0\end{cases}
\end{gathered}
$$

with

$$
\varphi(z)=\sum_{i=1}^{3} \gamma_{i}\left(\chi-\frac{\lambda_{3}}{\lambda_{i}}\right) w_{i(k-1)}-\frac{2 Q}{\alpha} z+q,
$$

where the root of the equation $\varphi(z)=0$ is

$$
z^{*}=\frac{\alpha}{2 Q}\left[\sum_{i=1}^{3} \gamma_{i}\left(\chi-\frac{\lambda_{3}}{\lambda_{i}}\right) w_{i(k-1)}+q\right] .
$$

Here, $z_{k}=t_{k} / a-k+1$ is the relative pulse duration for the control pulse $\mathrm{K}^{(\mathrm{F})}(\xi)\left(0 \leq z_{k} \leq(1 / 2)\right)$.

The period $T$ of the periodic motion of the dynamical system (1) is multiple to the external action period, i.e., $T=m a, m=$ $1,2, \ldots$ Here and hereinafter, we denote the motion with such a period as an $m$ cycle [17], [25], and [26].

Using the recurrence relations (3) as did Baushev and Zhusubaliyev [17] and [25], and the periodicity conditions $w_{i 0}=w_{i m}$, one obtains the expression for $w_{i k}$

$$
\begin{aligned}
w_{i k}= & \frac{1}{1-\mathrm{e}^{m a \lambda_{i}}}\left[\sum_{i=k+1}^{m} \mathrm{e}^{a \lambda_{i}(m+k-i)}\left(\mathrm{e}^{a \lambda_{i}\left(1-z_{i}\right)}-\mathrm{e}^{a \lambda_{i} z_{i}}\right)\right. \\
& \left.+\sum_{i=1}^{k} \mathrm{e}^{a \lambda_{i}(k-i)}\left(\mathrm{e}^{a \lambda_{i}\left(1-z_{i}\right)}-\mathrm{e}^{a \lambda_{i} z_{i}}\right)\right]+1
\end{aligned}
$$

Substituting $w_{i(k-1)}$ into (5) for $\varphi(z)$, we find

$$
\begin{aligned}
\varphi_{k}\left(z_{1}, \ldots, z_{m}\right) & =\sum_{i=1}^{3} \gamma_{i}\left(\chi-\frac{\lambda_{3}}{\lambda_{i}}\right) \sigma_{i k}-\frac{2 Q}{\alpha} z_{k}+q, \\
k & =\overline{1, m}
\end{aligned}
$$


where

$$
\begin{aligned}
\sigma_{i k}= & \frac{1}{1-\mathrm{e}^{m a \lambda_{i}}}\left[\sum_{i=k}^{m} \mathrm{e}^{a \lambda_{i}(m+k-1-i)}\left(\mathrm{e}^{a \lambda_{i}\left(1-z_{i}\right)}-\mathrm{e}^{a \lambda_{i} z_{i}}\right)\right. \\
& \left.+\sum_{i=1}^{k-1} \mathrm{e}^{a \lambda_{i}(k-1-i)}\left(\mathrm{e}^{a \lambda_{i}\left(1-z_{i}\right)}-\mathrm{e}^{a \lambda_{i} z_{i}}\right)\right]+1 .
\end{aligned}
$$

Using (4), one obtains the system of transcendental equations with respect to $z_{k}, k=\overline{1, m}[17]$, [25]

$$
\begin{gathered}
\varphi_{k}\left(z_{k}, z_{1}, \ldots, z_{m}\right)=\mu_{k}\left(z_{k}\right), k=\overline{1, m} \\
\mu_{k}\left(z_{k}\right)= \begin{cases}0, & 0<z_{k}<1 \\
>0, & z_{k}=1 \\
<0, & z_{k}=0 .\end{cases}
\end{gathered}
$$

The above algorithm allows us to evaluate not only the stable $m$ cycles but the unstable ones as well. If $z_{k}$ and $k=\overline{1, m}$ are the solutions of the (7), then, $m$ cycle $w_{i k}, k=\overline{1, m}$ can be evaluated according to (6).

The local stability of an $m$ cycle is determined by the eigenvalues of the basic matrix $F(T)$ which are the roots of

$$
\operatorname{det}(F(T)-\rho E)=0
$$

where the basic matrix can be determined by the recurrent formula

$$
\begin{aligned}
& F_{k}=\frac{\mathrm{d} W_{k}}{\mathrm{~d} W_{k-1}} F_{k-1}, k=\overline{1, m} \\
& F_{0}=E ; F(T)=F_{m} ; W=\left(w_{1}, w_{2}, w_{3}\right)^{\mathrm{T}}
\end{aligned}
$$

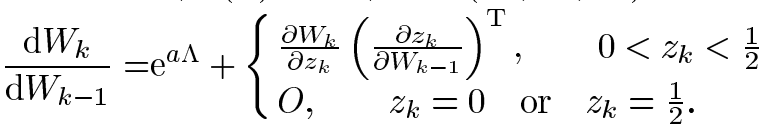

$$
\begin{aligned}
& \mathrm{e}^{a \Lambda}=\left(\begin{array}{ccc}
\mathrm{e}^{a \lambda_{1}} & 0 & 0 \\
0 & \mathrm{e}^{a \lambda_{2}} & 0 \\
0 & 0 & \mathrm{e}^{a \lambda_{3}}
\end{array}\right) \\
& \frac{\partial W_{k}}{\partial z_{k}}=\left(\begin{array}{l}
-a \lambda_{1}\left(\mathrm{e}^{a \lambda_{1}\left(1-z_{k}\right)}+\mathrm{e}^{a \lambda_{1} z_{k}}\right) \\
-a \lambda_{2}\left(\mathrm{e}^{a \lambda_{2}\left(1-z_{k}\right)}+\mathrm{e}^{a \lambda_{2} z_{k}}\right) \\
-a \lambda_{3}\left(\mathrm{e}^{a \lambda_{3}\left(1-z_{k}\right)}+\mathrm{e}^{a \lambda_{3} z_{k}}\right)
\end{array}\right) \\
& \frac{\partial z_{k}}{\partial W_{k-1}}=\frac{\alpha}{2 U_{0}}\left(\begin{array}{c}
\gamma_{1}\left(\chi-\frac{\lambda_{3}}{\lambda_{1}}\right) \\
\gamma_{2}\left(\chi-\frac{\lambda_{3}}{\lambda_{2}}\right) \\
\gamma_{3}(\chi-1)
\end{array}\right) \text {. }
\end{aligned}
$$

Here, $z_{k}$, where $k=\overline{1, m}$ is the root of (7), and $O$ is the zero matrix.

\section{BIFURCATION PHENOMENA IN DYNAMICS OF THE DC-DC CONVERTER}

\section{A. Subcritical Torus Bifurcation and Hysteretic Phenomena}

As previously noted, we choose the amplification constant $\alpha$, and transfer constant $\chi$ of the corrector as control parameters. Fig. 3 shows the arrangement of the domains of existence of various stable cycles of the map (3), (4) in the plane $(\alpha, \chi)$. Let us examine how the dynamics of the map (3) and (4) change if the parameters $\alpha$ and $\chi$ are varied within the limits indicated in the diagram (Fig. 3).

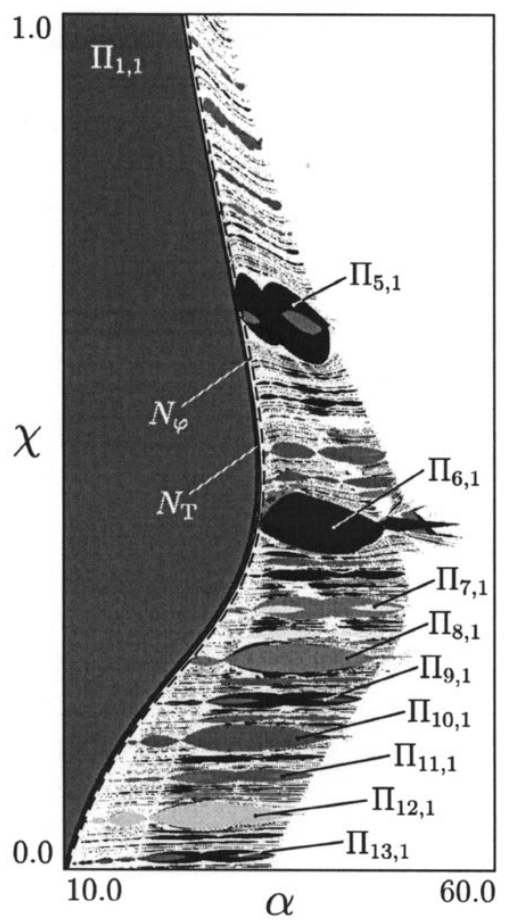

Fig. 3. Domains of existence for various dynamical modes of the maps (3) and (4) within the plane of control parameters $(\alpha, \chi) . \alpha$ and $\chi$ are the amplification and transfer constants of the PI corrector, respectively.

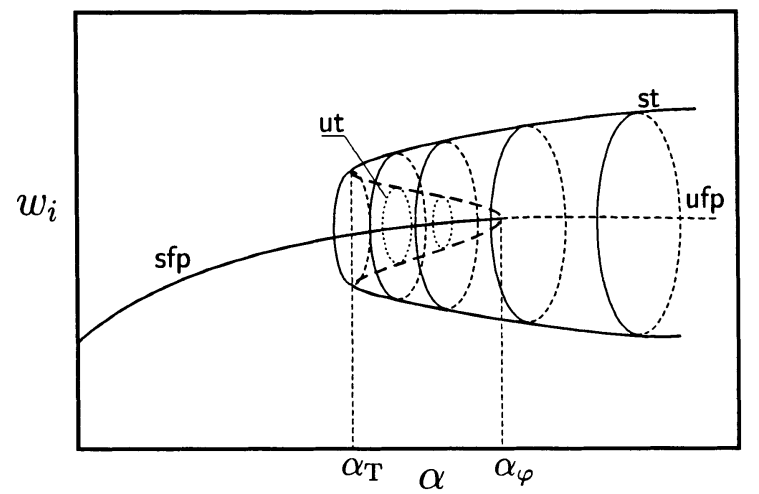

Fig. 4. Subcritical bifurcation of birth of a 2-D torus, where sfp is the stable fixed point, ut is the unstable torus, st is the stable torus, and ufp is the unstable fixed point.

When $\alpha$ is small enough, the system displays a globally stable period- 1 cycle (the operating mode). Its domain of existence is denoted as $\Pi_{1,1}$ in Fig. 3 . With increasing $\alpha$, the period- 1 cycle loses its stability through a subcritical Andronov-Hopf (or torus birth) bifurcation along the dashed curve $N_{\varphi}$ (which is the right boundary of $\left.\Pi_{1,1}\right)$. A sketch of this transition is presented in Fig. 4. Two 2-D tori appear at the point $\alpha_{\mathrm{T}} \in N_{\mathrm{T}}$, one of which is attracting, while the other is repelling. The latter merges with the fixed point in the point $\alpha_{\varphi} \in N_{\varphi}$ and disappears as the fixed point turns into an unstable focus. Thus, in the domain between $\alpha_{\mathrm{T}}$ and $\alpha_{\varphi}$ (i.e., between the curves $N_{\mathrm{T}}$ and $N_{\varphi}$ ), the stable fixed point coexists with attracting and repelling tori (see Fig. 5). Hard transitions take place at the boundaries of the domain between the curves $N_{\mathrm{T}}$ and $N_{\varphi}$, from quasi-periodic oscillations to the fixed point when $\alpha \in N_{\mathrm{T}}$, and from the fixed point to quasi-periodic oscillations when $\alpha \in N_{\varphi}$. 

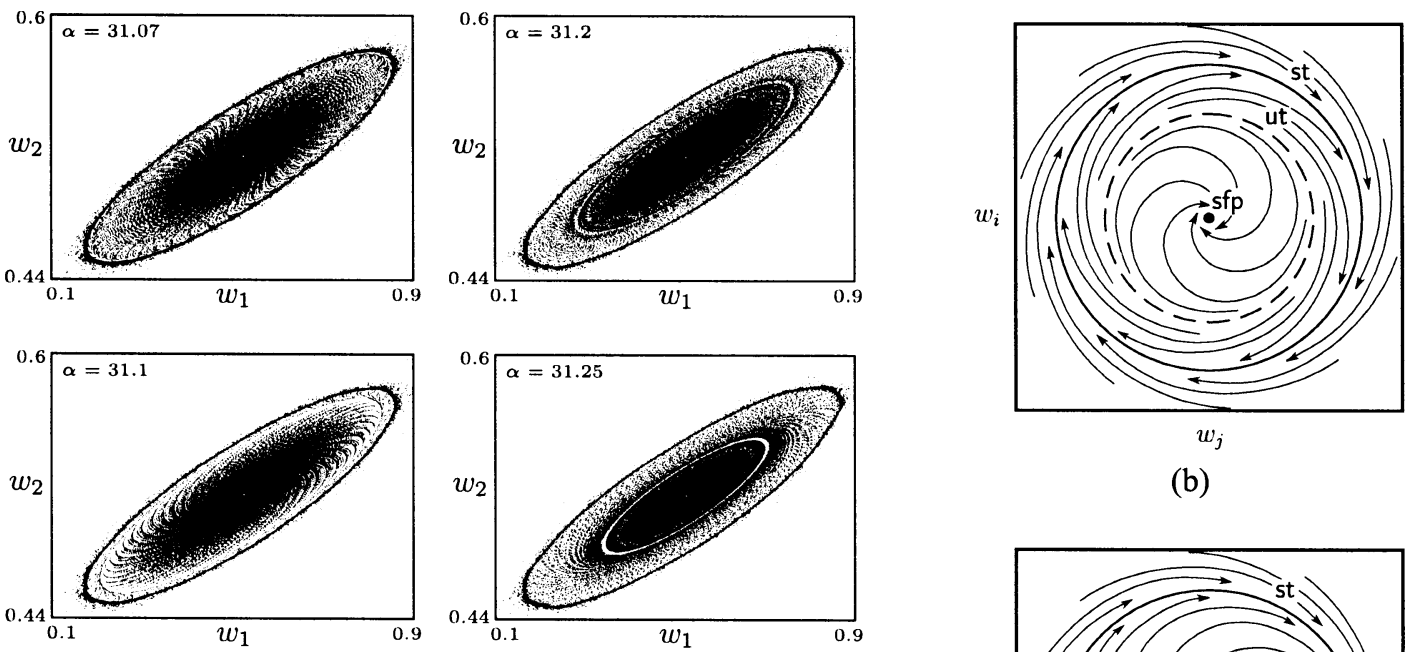

(b)

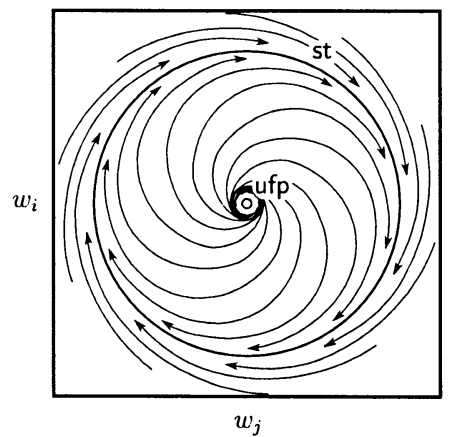

(c)

(a)

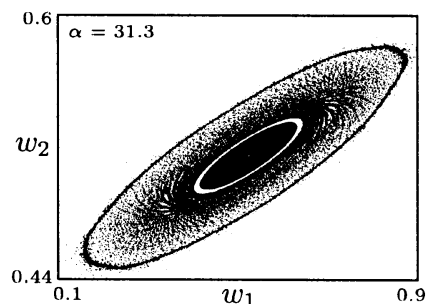

Fig. 5. Phase portraits of the Poincaré map within the domain between the curves $N_{\mathrm{T}}$ and $N_{\varphi}$. (a) Results of numerical experiments for $\chi=0.35$ and values of $\alpha$ increasing from $\alpha=\alpha_{\mathrm{T}}$ to $\alpha=\alpha_{\varphi}\left(\alpha_{\mathrm{T}} \approx 31.0615\right.$ and $\left.\alpha_{\varphi} \approx 31.40688\right)$. The situation of the unstable torus whose diameter decreases toward zero with increasing $\alpha$ is easily seen on the diagrams. (b) Sketch of the phase portrait for $\alpha_{\mathrm{T}}<\alpha<\alpha_{\varphi}$, where sfp is the stable fixed point, ut is the unstable torus, and st is the stable torus. (c) Sketch of the phase portrait for $\alpha>\alpha_{\varphi}$, where ufp is the unstable focus point.

Within the range $\left[\alpha_{\mathrm{T}}, \alpha_{\varphi}\right]$ (i.e., between the curves $N_{\mathrm{T}}$ and $N_{\varphi}$ ) external disturbances, that are always present in operating systems, may lead to hard transitions from the quasi-periodic mode to the period- 1 cycle and vice versa, depending on the basin of attraction that contains the trajectory after the disturbance has acted. The boundary between the basins of the quasiperiodic mode and of the fixed point is formed by the repelling torus (see Fig. 5(a) and (b)). When $\alpha$ increases from $\alpha_{\mathrm{T}}$ to $\alpha_{\varphi}$, the diameter of the repelling torus decreases from some initial value to zero. Thus, if we consider the period- 1 cycle as the operating mode and the transition to quasi-periodic oscillations as a catastrophe, then the probability of such a catastrophe is equal to zero at $\alpha<\alpha_{\mathrm{T}}$ (to the left of $N_{\mathrm{T}}$ ), equal to one at $\alpha>\alpha_{\varphi}$ (to the right of $N_{\varphi}$ ), and smoothly increases from 0 to 1 , as $\alpha$ increases from $\alpha_{\mathrm{T}}$ to $\alpha_{\varphi}$ (moves between $N_{\mathrm{T}}$ and $N_{\varphi}$ ).

Intersection of domains of existence for different modes leads to the appearance of hysteretic phenomena in the dynamics. Fig. 6 shows bifurcation diagrams for the interval $\left[\alpha_{\mathrm{T}}, \alpha_{\varphi}\right]$ for different scanning directions of the parameter $\alpha$ : Fig. 6(a) for increasing and Fig. 6(b) for decreasing value of $\alpha$.

We conclude that the system can operate in different modes with the same values of the parameters depending on the trajectory of deformation that leads to this point in the parameter plane.

With further increase of $\alpha$, resonances on the 2-D torus occur. The nature of the boundaries of resonance domains and the bifurcations within these domains are discussed in the next section.

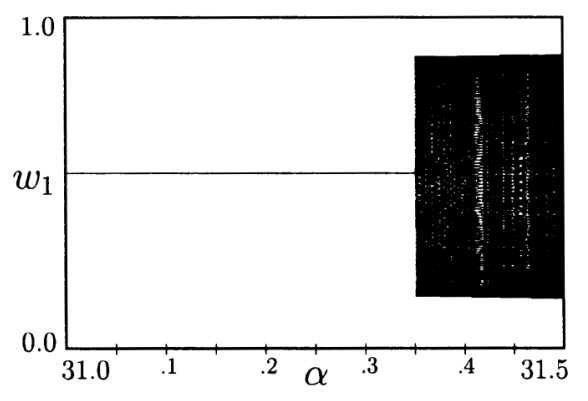

(a)

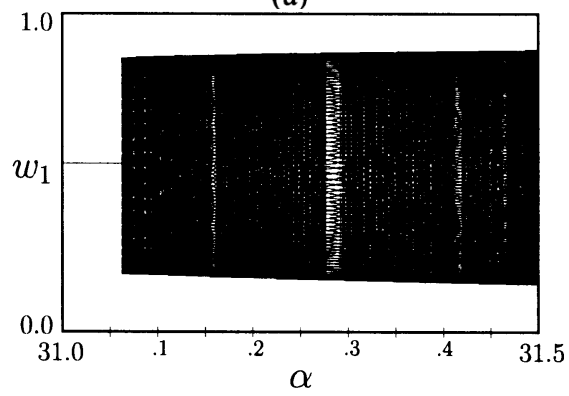

(b)

Fig. 6. Transitions near the Andronov-Hopf bifurcation point $\alpha_{\varphi}$ and the point $\alpha_{\mathrm{T}}$, where the stable and unstable tori merge: (a) when $\alpha$ increases; (b) when $\alpha$ decreases.

\section{B. BCB on a 2-D Torus and Transitions to Chaos}

In our previous work [16], [17], we described the detailed inner structure of the resonance domains and the nature of the bi- 
furcation boundaries for a dc-dc converter with one-sided PWM of the first kind. We will briefly review these results, because they remain valid for the system under consideration.

Within the parameter plane $\Pi=\{(\alpha, \chi)\}$ we discovered domains of existence of resonance cycles on a 2-D torus (domains of synchronization) which have a peculiar shape. These domains are similar to the Arnol'd tongues described in numerous works for maps as well as for flow systems [30]-[34]. Domains of synchronization for piecewise-smooth systems, however, exhibit important differences with respect to their inner structure and bifurcational transitions.

Our numerical analysis shows that the tongues of synchronization are located everywhere dense within the parameter plane. Nevertheless, their measure close to the curve where the 2-D torus arises from a period-1 cycle can be very small. To the right of this curve, the total width first increases, but nonmonotonically, and then decreases, also nonmonotonically. Moreover, the resonance tongues are bounded not by the curves of saddle-node bifurcation, like for smooth dynamical systems (see, e.g., [30]-[34]), but by lines where BCB occur. Between the synchronization tongues there are domains of quasi-periodicity and chaos. Within the individual tongue, a finite sequence of $\mathrm{BCB}$ of resonance cycles is observed, leading at the end to chaotic dynamics.

Let us denote the simply connected domains of existence of cycles within the plane of control parameters $\Pi$ as $\Pi_{m, k}$, $\Pi_{m, k} \subset \Pi$; where $m$ is the period of cycle and index $k$ serves to distinguish domains with the same $m$.

The synchronization domains may be divided into two groups. The criterion for this division is the difference in their inner structure and the bifurcations of the resonance cycles. The first group comprises such tongues $\Pi_{m, k}$ within which there is a finite number of simply connected domains $\hat{\Pi}_{m, k}$, $\hat{\Pi}_{m, k} \subset \Pi_{m, k}$ where two stable resonance cycles of different type coexist. When intersecting the boundaries of the domains $\hat{\Pi}_{m, k}$ within such tongues one can observe hard hysteretic transitions from one stable $m$ cycle to another. The second group includes such $\Pi_{m, k}$ where, when the parameters change within $\Pi_{m, k}$, the transitions from one type of a stable resonance $m$ cycle to another take place in a smooth way via BCB involving a simple change of the type of solution. Tongues of the first group are the domains where the period of the resonance cycle is odd, while those of the second group have even periods.

Figs. 7 and 8 show the shape and the boundary structures for two synchronization tongues taken as an example. These are the domains of existence for the stable resonance period-11 cycle (shown in Fig. 7) and for the stable resonance period-10 cycle (shown in Fig. 8) that belong to the first and the second group, respectively. In Fig. 7, the shaded regions are the domains of bistability where two stable period-11 resonance cycles of different types, coexist.

Let us consider the main BCB boundaries. These are the lines denoted in Figs. 7 and 8 as $L_{1}, L_{2}, L_{3}$, and $L_{4}$. Each of these lines is a union of BCB boundaries of two kinds

$$
L_{k}=\bigcup_{i} L_{i, k}^{ \pm}, k=\overline{1,4}
$$

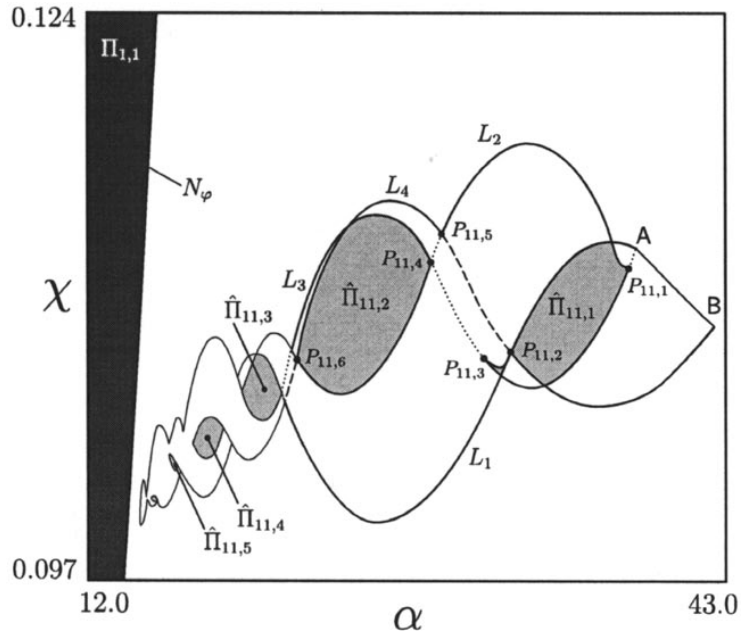

Fig. 7. Domain of stability of period-11 cycle $\Pi_{11,1}$.

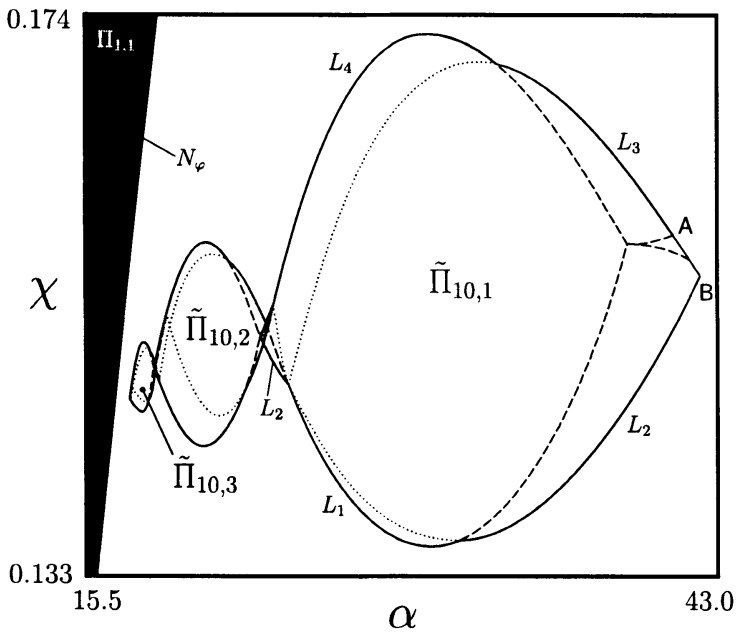

Fig. 8. Domain of stability of period-10 cycle $\Pi_{10,1}$.

The curves $L_{i, k}^{+}$correspond to the boundaries $N_{+}^{\mathrm{C}}$ where stable and unstable cycles of different types merge and disappear, and $L_{i, k}^{-}$correspond to the boundaries with a simple change of the solution type.

The resonance domains are bounded by an aggregate of $\mathrm{BCB}$ lines of the type $L_{i, k}^{+}$, with the exception of the segment $\mathrm{AB}$, which is a boundary of type $N_{-}^{\mathrm{C}}$, where the solution changes its type with loss of stability.

The difference between resonance domains from the first and second group first of all lies in the presence of domains of coexistence of two stable $m$ cycles of different types. In Fig. 7, such domains are denoted as $\hat{\Pi}_{11, i}, \hat{\Pi}_{11, i} \subset \Pi_{11,1}, i=1,2, \ldots$. The domains $\hat{\Pi}_{11, i}$ are bounded by the BCB lines $L_{i, k}^{+}$in the points of which a stable period-11 cycle merges with an unstable one of another type and disappears. Within each $\hat{\Pi}_{11, i}$ periodic motions smoothly depend upon the parameters, while intersection of the BCB boundaries $L_{i, k}^{+}$results in hard transitions from a stable period- 11 cycle to a stable period-11 cycle of another type and vice versa, with hysteresis as one typically observes for such transitions. The bifurcation lines $L_{i, k}^{+}$within $\Pi_{11, i}$ are supported by the points $P_{11, i}, i=1,2, \ldots$ of codimension two. 


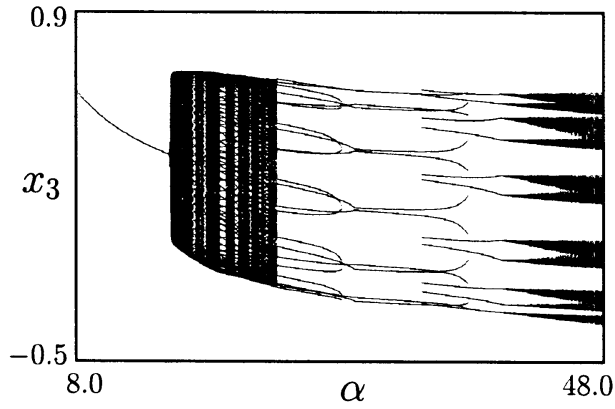

(a)

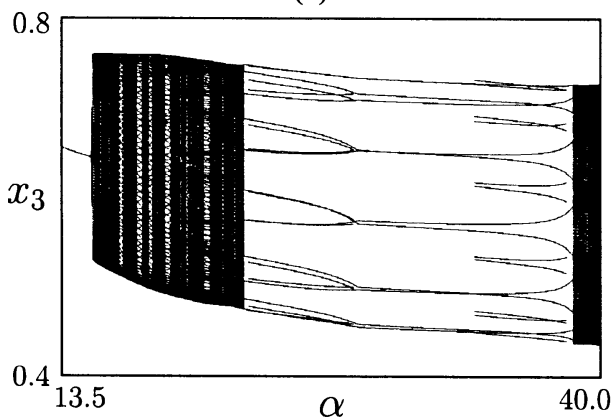

(b)

Fig. 9. Bifurcations within $\Pi_{11,1}$. (a) Soft appearance of a cycle of chaotic intervals. (b) Transition to quasi-periodic mode.

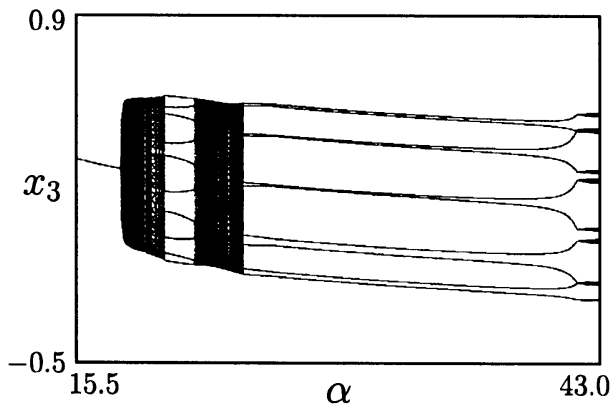

Fig. 10. Bifurcations within $\Pi_{10,1}$.

The dashed and dotted lines in Fig. 7 mark the boundaries where a simple change of the number of the trajectory portions being sewed occurs (a simple change of the solution type [3]). They are continuation of the BCB boundaries $L_{i, k}^{+}$as represented by solid lines in the diagram. If the parameters are changed along the curve $L_{k}, k=\overline{1,4}$, this means that the curves $L_{i, k}^{+}$continuously convert into the BC-bifurcation curves $L_{i, k}^{-}$ of a simple change of the type of solution when passing through the points $P_{11, i}, i=1,2, \ldots$ of codimension two. Dotted lines show the BCB boundaries for the change of the type of solution for the stable cycles, and the dashed lines show the same for unstable cycles.

When moving from the domain $\hat{\Pi}_{11, i}$ into the domain $\hat{\Pi}_{11, i+1}$, the number of parts from which the trajectory of a stable period- 11 cycle is sewed successively, increases in steps of two. For example, the trajectories of the stable cycles existing in $\hat{\Pi}_{11,1}$ is sewed from four parts, in $\hat{\Pi}_{11,2}$ from six, etc., and in $\hat{\Pi}_{11,9}$ their number is 20 . The number of parts from which the phase trajectory of a period- 11 cycle is sewed is

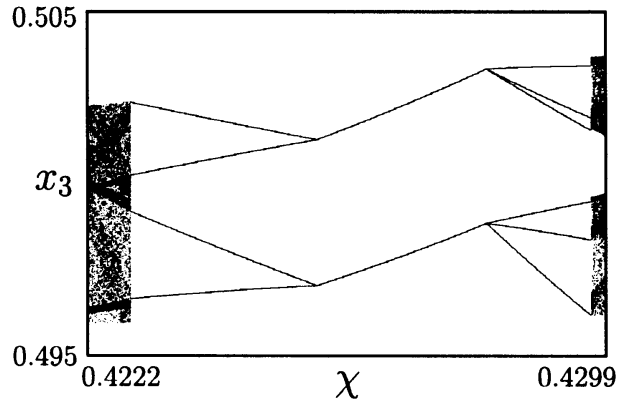

Fig. 11. Doubling and tripling of the cycle period on the boundaries of resonance domains. This diagram contains only two branches of six (the cycle period is equal to 6 ).

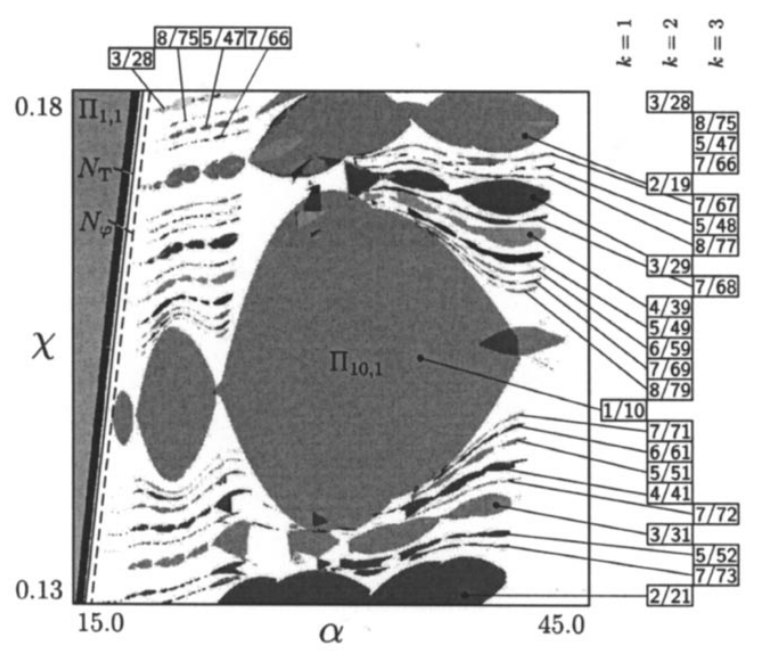

(a)

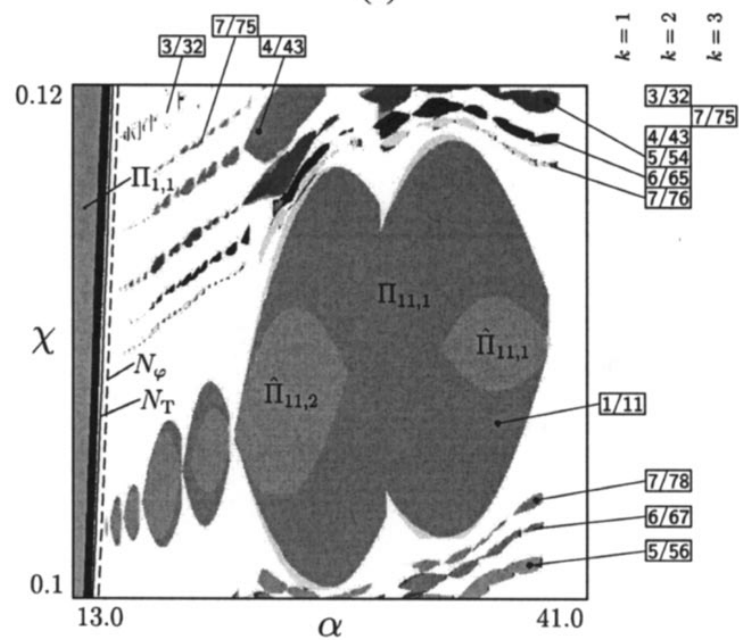

(b)

Fig. 12. Resonance cycles of the first, second, and third level of complexity $k$.

limited (it cannot exceed 22). Hence, the number of domains $\hat{\Pi}_{11, i}$ is finite.

The inner structure of the synchronization domains for the second group is simpler than that for the first group. The parts of the lines $L_{k}$ in Fig. 8 that correspond to $\mathrm{BCB}$ of a simple change of the solution type of the stable and unstable period- 10 cycle are inner ones and divide the domain $\Pi_{10,1}$ into four parts. In each of these regions, a single stable cycle of a particular 

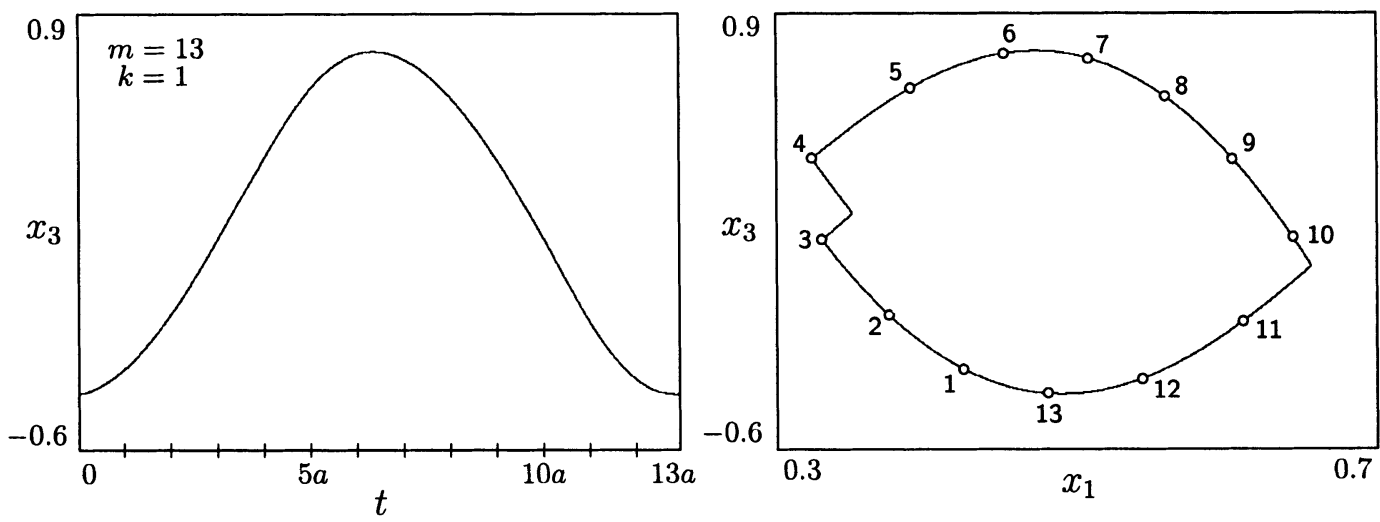

(a)
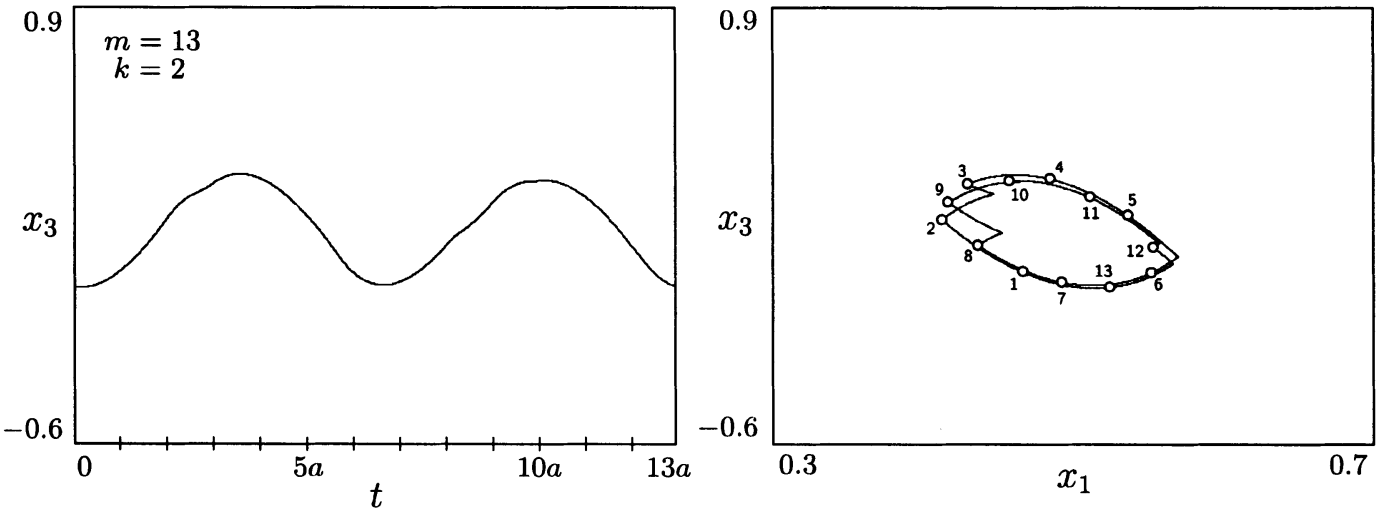

(b)

Fig. 13. Time series for the state variable $x_{3}$ for the one oscillation period (left) and 2-D projections of the integral curves (right) for the period-13 cycle: (a) 1-turned cycle $(\alpha=26.9, \chi=0.017$, rotation number $=1 / 13$, level of complexity $=1)$; (b) 2 -turned cycle $(\alpha=38.0 ; \chi=0.345$, rotation number $=$ $2 / 13$, level of complexity $=2$ ). Circles on the integral curve mark the positions of the Poincaré map points, the numbers indicating the sequence of the iterations.

type exists. Within every $\tilde{\Pi}_{10, i}, i=1,2, \ldots$, the number of which inside $\Pi_{10,1}$ is finite, stable cycles of four different types exist. When changing the parameters, a stable resonance cycle continuously transforms into a stable cycle of another type. This happens in the points of the BC-boundaries marked in Fig. 8 by the dashed lines.

Now let us consider the changes in the system behavior at the intersecting bifurcation boundaries.

When intersecting the boundary $N_{+}^{\mathrm{C}}$, a stable resonance cycle disappears as it merges with an unstable cycle of a different type. Hard transitions to a resonance cycle with another period or to chaotic oscillations developed form a resonance cycle with another period are possible here. Such transitions occur within the domain of resonance overlap.

When intersecting that part of the boundaries of $\Pi_{10,1}$ or $\Pi_{11,1}$ which corresponds to the line $N_{-}^{\mathrm{C}}$ (the segments $\mathrm{AB}$ in Figs. 7 and 8), a resonance cycle changes its type and becomes a saddle. Here, a cycle of chaotic intervals softly arises from the resonance cycle. As shown in our previous work [15], the skeleton of this cycle of chaotic intervals is a denumerable set of saddle cycles with periods that are multiple to the period of the basic cycle.

One of the possible transitions to chaos via a sequence of $\mathrm{BCB}$ within the resonance domains of the first kind is illustrated by the bifurcation diagram depicted in Fig. 9(a). With change of the parameters a resonance cycle undergoes both hysteresis and soft $\mathrm{BCB}$ transitions. When intersecting the line $\mathrm{AB}$, a cycle of chaotic intervals softly arises from a stable cycle via a border-collision bifurcation. Another bifurcation scenario when changing the parameters within such domains is illustrated in Fig. 9(b). This diagram is obtained for the system considered in this paper. Here, the transition from periodic to quasi-periodic oscillations occurs abruptly when two period-11 cycles of different types merge and disappear at the boundary $N_{+}^{\mathrm{C}}$.

Bifurcations of a resonance cycle and transitions to chaos which are related with $\Pi_{10,1}$ are on the whole similar to those of $\Pi_{11,1}$. The only exception is that hysteresis transitions do not occur (see Fig. 10).

There are other kinds of resonance domains within $\Pi$ that display certain differences in the structure of their bifurcation boundaries by comparison with the above considered domains. These differences lie in the presence of BCB lines of resonance cycle period-doubling and tripling within the synchronization tongues (see, for example, Fig. 11). A detailed study of bifurcational transitions in the points of such curves was performed in our previous work [15], [17].

\section{Arrangement of the Resonance Domains Within the Parameter Plane}

Domains of stability of resonance cycles are arranged within the parameter plane in such a way, that their rotation numbers are ordered according to the well-known Farey tree [41]. This means that the rotation number of such a cycle can be repre- 

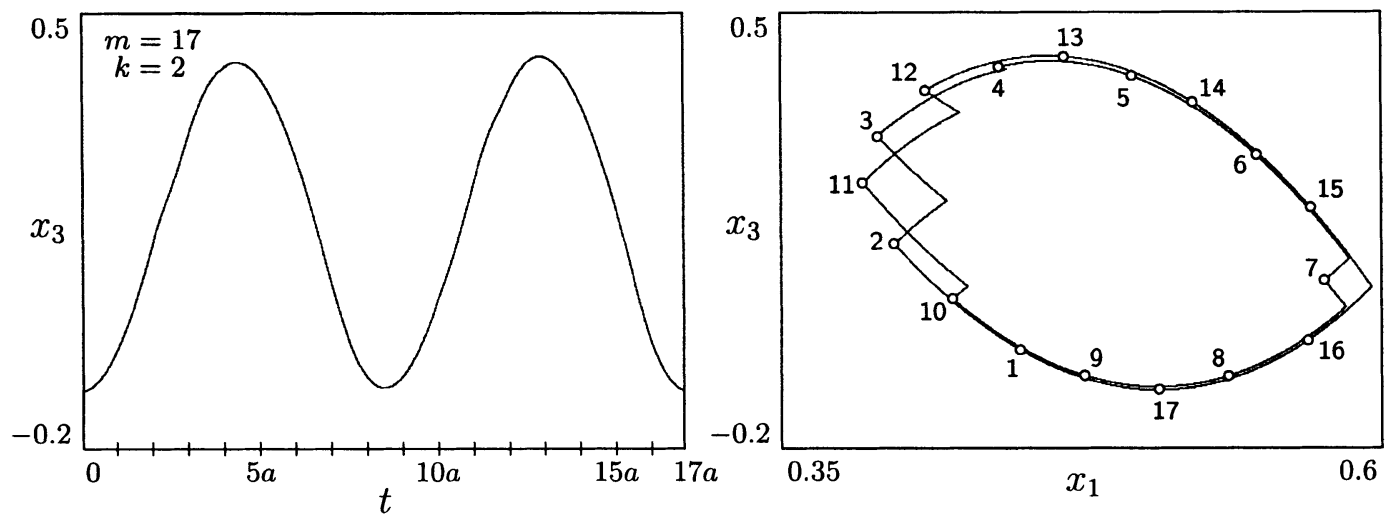

(a)
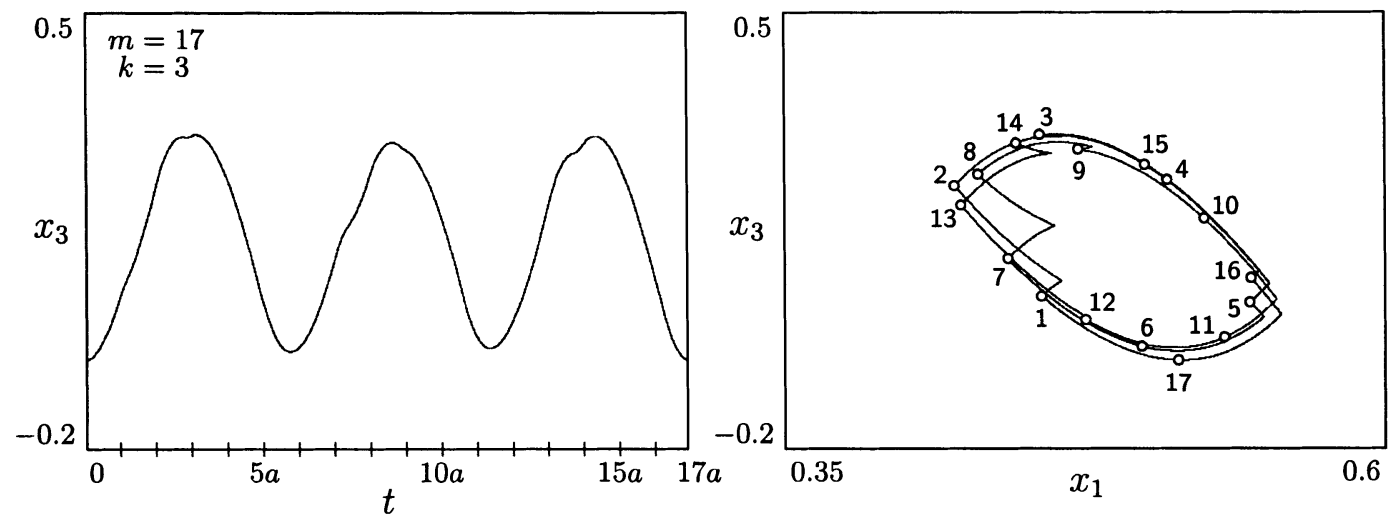

(b)
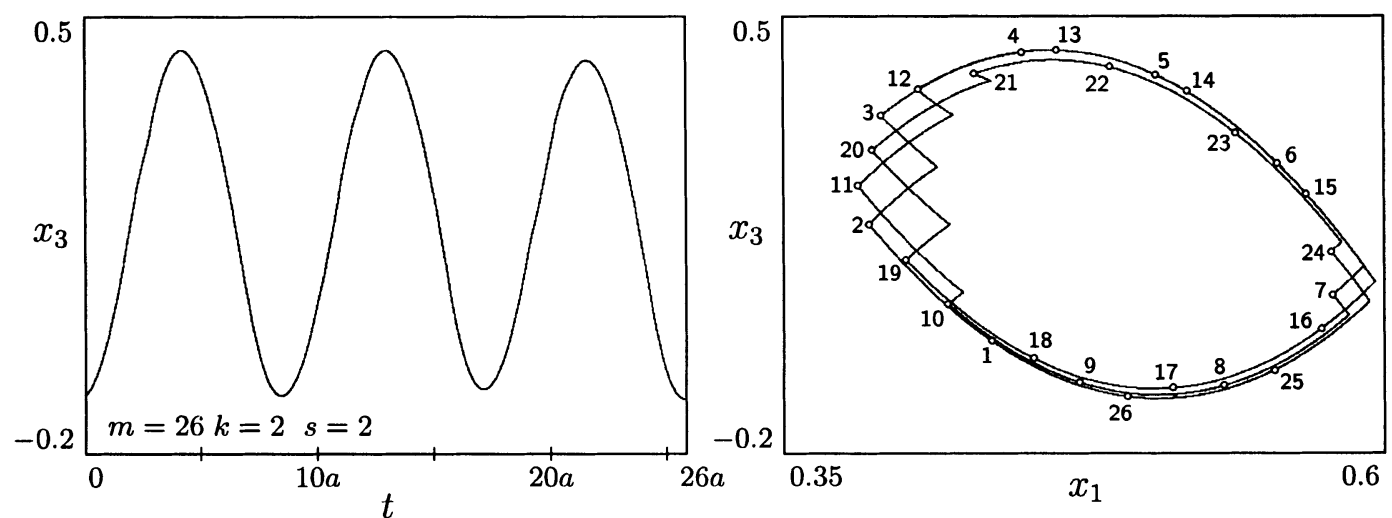

(c)

Fig. 14. Time series for the state variable $x_{3}$ for the one oscillation period (left) and 2-D projections of the integral curves (right) for: (a) 2-turned period-17 cycle $(\alpha=31.0, \chi=0.22$, rotation number $=2 / 17$, level of complexity $=2)$; (b) 3-turned period-17 cycle $(\alpha=37.0, \chi=0.46$, rotation number $=3 / 17$, level of complexity $=3)$; (c) 3-turned period-26 cycle $(\alpha=29.9, \chi=0.21$, rotation number $=3 / 26$, level of complexity $=2, s=2)$. Circles on the integral curve mark the positions of the Poincaré map points, the numbers indicating the sequence of the iterations.

sented as

$$
\frac{r_{k}^{s}}{q_{k}^{s}}=\frac{s r_{k-1}^{m}+r_{k-1}^{m+1}}{s q_{k-1}^{m}+q_{k-1}^{m+1}}
$$

or

$$
\frac{r_{k}^{s}}{q_{k}^{s}}=\frac{r_{k-1}^{m}+s r_{k-1}^{m+1}}{q_{k-1}^{m}+s q_{k-1}^{m+1}} .
$$

Here, $k=1,2, \ldots$ is the level of complexity [42]; $s=1,2, \ldots$ is the number of the cycle in the sequence of cycles of $k$ th level of complexity; $r_{k-1}^{m} / q_{k-1}^{m}$ and $r_{k-1}^{m+1} / q_{k-1}^{m+1}$ are the rotation num- bers of the nearest neighboring cycles of $(k-1)$ th level of complexity; $r_{1}^{m}=1$ and $q_{1}^{m}=m+1$.

In other words, the Farey principle for the case under consideration can be stated as follows: between any two domains of existence of stable resonance cycles with the rotation numbers $a / b$ and $c / d$ one always finds the domain of existence of a stable resonance cycle with the rotation number

$$
\frac{r}{q}=\frac{a}{b} \oplus \frac{c}{d}=\frac{a+c}{b+d} \text {. }
$$

Fig. 12 shows the arrangement of resonance domains of various levels of complexity within the plane of control parameters. 
Let us consider the form of the rotation number for multidimensional dynamical systems.

(a) The denominator of the rotation number determines the oscillation period. In particular, for control systems with PWM (1) if $r / q$ is the rotation number of a resonance cycle, then the period of this cycle is $T=q a$, where $a$ is the period of modulation. The period of oscillation for the Poincaré map equals $q$.

As long as we only consider the period of the cycle the operation " $\oplus$ " can be replaced with common addition while building the Farey series, i.e., between two resonance domains one always finds a domain with a cycle period that is equal to the sum of the cycle periods of the original domains.

(b) The numerator of the rotation number determines how many turns along the big circle of the torus the trajectory will perform during the period, i.e., the resonance cycle with the rotation number $r / q$ rotates $r$ times in $q$ iterations.

All the discussed regularities are also valid for control systems with one-sided PWM of the first kind as described in our previous work [16]. As an illustrative example, Figs. 13 and 14 show the trajectories for cycles with the same periods, but different rotation numbers for this system. Note the succession of the Poincare map iterations for the cycles with different numerator of the rotation number. These figures clearly show that for the given period the big diameter of the torus changes inversely proportional to the numerator of the rotation number. This can be explained as follows: the rate of the state variable changes is basically determined by the values of the reactive elements and remains nearly constant.

Fig. 14(c) is presented to avoid the false impression that the number of turns that the trajectory performs during one period is determined by the level of complexity. There are cycles with the same periods and the same level of complexity but with different numerator of the rotation number, for example, period-57 cycles of the second level of complexity. The domain of existence of one such cycle lies between $\Pi_{7,1}$ and $\Pi_{8,1}$, and the rotation number $r / q=8 / 57$ (see (8) for $k=2, m=6, s=7$ ). For another period-57 cycle the domain of existence lies between $\Pi_{8,1}$ and $\Pi_{9,1}$, and $r / q=7 / 57$ (see (8) for $k=2, m=7, s=6$ ). Generally, it should be noted that increase of the numerator of the rotation number leads to an increase of the complexity of motion even if the motion period (denominator of the rotation number) does not change. In practical applications this results in an increasing probability for the occurrence of incoherent motions due to jumping of the phase point from one part of the integral curve to another [43].

\section{CONCLUSION}

We studied the bifurcation phenomena that can be observed in a dc-dc voltage converter with two-sided PWM. The amplification constant $\alpha$ and the transfer constant $\chi$ for the PI-corrector were used as bifurcation parameters. By contrast to the case of one-sided PWM [16], the system displays a subcritical transition to quasi-periodicity. This implies that there is a parameter interval in which a stable periodic state (the operating mode) coexists with a stable and an unstable torus. The basin of attraction for the operating mode may be very small, however, so that in practice the system can be unstable.

A similar phenomenon was recently described by Christiansen et al. [44] for the so-called transonic flutter in aircraft wings, i.e., the self-excited excitations of the elastic modes of the wing structure that can arise through interaction with the aerodynamical flow. These authors also investigated how random fluctuations in the airflow could induce transitions between the stable operating mode and finite amplitude flutter oscillations.

In the regime of quasi-periodicity, both the one-sided and the two-sided pulsewidth modulated voltage converter display transitions between resonant and nonresonant behavior. By contrast to the well-established theory for smooth dynamical systems [45], these transitions take place via BCB rather than via saddle-node bifurcation. Moreover, the inner organization of the resonance domains is also quite different from the case of smooth dynamical systems. We suggest that the transition to chaos through torus destabilization can also occur via mechanisms that differ from the mechanisms known for smooth dynamical systems [29] and [46].

\section{REFERENCES}

[1] M. I. Feigin, "Doubling of the oscillation period with C-bifurcations in piecewise continuous systems" (in Russian), Prikl. Mat. Mekh., vol. 34, no. 5, pp. 861-869, 1970.

[2] _ _ "On the generation of sets of subharmonic modes in a piecewise continuous system" (in Russian), Prikl. Mat. Mekh., vol. 38, no. 5, pp. 810-818, 1974.

[3] - Forced Oscillations in Systems with Discontinuous Nonlinearities (in Russian). Moscow, Russia: Nauka, 1994.

[4] — "The increasingly complex structure of the bifurcation tree of a piecewise-smooth system," J. Appl. Math. Mech., vol. 59, pp. 853-863, 1995.

[5] H. E. Nusse and J. A. Yorke, "Border-collision bifurcations including "period two to period three" for piecewise smooth systems," Physica, vol. D57, pp. 39-57, 1992.

[6] _ , "Border-collision bifurcation for piecewise smooth one-dimensional maps," Int. J. Bifurcation Chaos, vol. 5, no. 1, pp. 189-207, 1995.

[7] H. E. Nusse, E. Ott, and J. A. Yorke, "Border-collision bifurcations: An explanation for observed bifurcation phenomena," Phys. Rev., vol. E49, pp. 1073-1076, 1994.

[8] M. di Bernardo, M. I. Feigin, S. J. Hogan, and M. E. Homer, "Local analysis of C-bifurcations in $n$-dimensional piecewise-smooth dynamical systems," Chaos, Solitons Fractals, vol. 10, no. 11, pp. 1881-1908, 1999.

[9] S. Banerjee, J. A. Yorke, and C. Grebogi, "Robust chaos," Phys. Rev. Lett., vol. 80, no. 14, pp. 3049-3052, 1998.

[10] S. Banerjee and C. Grebogi, "Border collision bifurcations in two-dimensional piecewise smooth maps," Phys. Rev. E, vol. 59, no. 4, pp. 4052-4061, 1999.

[11] S. Banerjee, M. S. Karthik, G. Yuan, and J. A. Yorke, "Bifurcations in one-dimensional piecewise smooth maps-Theory and applications in switching circuits," IEEE Trans. Circuits Syst. I, vol. 47, pp. 389-394, Mar. 2000.

[12] S. Banerjee, P. Ranjan, and C. Grebogi, "Bifurcations in two-dimensional piecewise smooth maps-Theory and applications in switching circuits," IEEE Trans. Circuits Syst. I, vol. 47, pp. 633-643, May 2000.

[13] M. di Bernardo, C. J. Budd, and A. R. Champneys, "Grazing and bordercollision in piecewise-smooth systems: A unified analytical framework," Phys. Rev. Lett., vol. 86, no. 12, pp. 2553-2556, 2001.

[14] - "Corner collision implies border collision," Physica D, vol. 154, no. 3-4, pp. 171-194, 2001.

[15] Z. T. Zhusubaliyev, E. A. Soukhoterin, and E. Mosekilde, "Border-collision bifurcations and chaotic oscillations in a piecewise-smooth dynamical system," Int. J. Bifurcation Chaos, vol. 11, no. 12, pp. 2977-3001, 2001.

[16] - "Border-collision bifurcations on a two-dimensional torus," Chaos, Solitons, Fractals, vol. 13, no. 9, pp. 1889-1915, 2002. 
[17] Z. T. Zhusubaliyev and E. Mosekilde, Bifurcations and Chaos in Piecewise-Smooth Dynamical Systems. Singapore: World Scientific, 2003.

[18] G. H. Yuan, S. Banerjee, E. Ott, and J. A. Yorke, "Border-collision bifurcations in the buck converter," IEEE Trans. Circuits Syst. I, vol. 45, pp. 707-716, July 1998 .

[19] M. di Bernardo and F. Vasca, "Discrete-time maps for the analysis of bifurcations and chaos in dc-dc conveters," IEEE Trans. Circuits Syst. I, vol. 47, pp. 130-143, Feb. 2000.

[20] M. di Bernardo and C. K. Tse, "Chaos in power electronics: An overview," in Chaos in Circuits and Systems, G. Chen and T. Ueta, Eds. Singapore: World Scientific, 2002, pp. 317-340.

[21] D. C. Hamill and D. J. Jefferies, "Subharmonics and chaos in a controlled switched-mode power converter," IEEE Trans. Circuits Syst., vol. 35, pp. 1059-1061, Aug. 1988.

[22] D. C. Hamill, J. H. B. Deane, and D. J. Jefferies, "Modeling of chaotic dc-dc conveters by iterated nonlinear mappings," IEEE Trans. Power Electron., vol. 7, pp. 25-36, Jan. 1992.

[23] A. E. Aroudi and R. Leyva, "Quasiperiodic route to chaos in a PWM voltage-controlled dc-dc boost converter," IEEE Trans. Circuits Syst. I, vol. 48, pp. 967-978, Aug. 2001.

[24] S. Banerjee and G. C. Verghese, Eds., Nonlinear Phenomena in Power Electronics. New York: IEEE Press, 2001.

[25] V. S. Baushev and Z. T. Zhusubaliyev, "Indeterminable states of a voltage regulator with pulsewidth control," Elect. Technol., no. 3, pp. 85-98, 1992.

[26] V. S. Baushev, Z. T. Zhusubaliyev, and S. G. Michal'chenko, "Stochastic features in the dynamic characteristics of a pulsewidth controlled voltage stabilizer," Elect. Technol., no. 1, pp. 137-150, 1996.

[27] E. Fossas and G. Olivar, "Study of chaos in the buck converter," IEEE Trans. Circuits Syst. I, vol. 43, pp. 13-25, Jan. 1996.

[28] A. E. Aroudi, L. Benadero, E. Toribio, and S. Machiche, "Quasiperiodicity and chaos in the dc-dc buck-boost converter," Int. J. Bifurcation Chaos, vol. 10, pp. 359-371, 2000.

[29] V. S. Afraimovich and L. P. Shil'nikov, "Invariant two-dimensional tori, their breakdown and stochasticity," Amer. Math. Soc. Transl., vol. 149, no. 2, pp. 201-212, 1991

[30] J. Maurer and A. Libchaber, "Rayleigh-benard experiment in liquid helium: Frequency locking and the onset of turbulence," J. Phys. Lett., vol. 40, pp. L419-L423, 1979 .

[31] D. G. Aranson, M. A. Chori, G. R. Hall, and R. P. McGenehe, "Bifurcations from an invariant circle for two-parameter families of maps of the plane: A computer-assisted study," Commun. Math. Phys., vol. 83, pp. 303-354, 1982

[32] V. Franceschini, "Bifurcations of tori and phase locking in a dissipative system of differential equations," Physica D, vol. 6, no. 3, pp. 285-304, 1982.

[33] J. Stavans, "Experimental study of quasi-periodicity in a hydrodynamical systems," Phys. Rev. A, vol. 35, pp. 4314-4328, 1987.

[34] V. S. Anishchenko and M. A. Safonova, "Mechanism of destruction of invariant curve in a model map of the plane" (in Russian), Radiotekh. Elektron., vol. 32, pp. 1207-1216, 1987.

[35] P. R. Severns and G. E. Bloom, Modern DC-to-DC Switchmode Power Converter Circuits. New York: Van Nostrand Reinhold, 1985.

[36] P. P. K. Chetty, Switch-Mode Power Supply Design. Blue Ridge Summit, PA: TAB Books Inc., 1986.

[37] B. K. Bose, Ed., Modern Power Electronics: Evolution, Technology, and Applications. New York: IEEE Press, 1992.

[38] Y. Z. Tsypkin, Sampling Systems Theory and Its Application. New York: Macmillan, 1964

[39] A. K. Gelig and A. N. Churilov, Stability and Oscillations of Nonlinear Pulse-Modulated Systems. Boston, : Birkhäuser, 1998.

[40] A. V. Kobzev, G. Y. Michal'chenko, and N. M. Muzhitchenko, Modulating Power Supplies of Radioelectronic Devices (in Russian). Tomsk, Russia: Radio i Svyaz, 1990

[41] B.-L. Hao, Elementary Symbolic Dynamics and Chaos in Dissipative Systems. Singapore: World Scientific, 1989.

[42] Y. L. Maistrenko, V. L. Maistrenko, S. I. Vikul, and L. O. Chua, "Bifurcations of attracting cycles from time-delayed Chua's circuit," Int. J. Bifurcation Chaos, vol. 5, no. 3, pp. 653-671, 1995.
[43] Z. T. Zhusubaliyev, E. A. Soukhoterin, V. N. Rudakov, Y. V. Kolokolov, and E. Mosekilde, "Bifurcations and chaotic oscillations in an automatic control relay system with hysteresis," Int. J. Bifurcation Chaos, vol. 11 no. 5, pp. 1193-1231, 2001.

[44] L. E. Christiansen, T. Lehn-Schiøler, E. Mosekilde, P. Gránásy, and H. Matsushita, "Nonlinear characteristics of randomly excited transonic flutter," Math. Comp. Sim., vol. 58, pp. 385-405, 2002.

[45] V. I. Arnol'd, "Small denominators. I. Mappings of the circumference into itself," AMS Trans. Series 2, vol. 46, pp. 213-284, 1965.

[46] V. I. Arnol'd, Ed., Dynamical Systems V, Encyclopaedia of Mathematical Sciences. Berlin, Germany: Springer-Verlag, 1991, vol. 5.

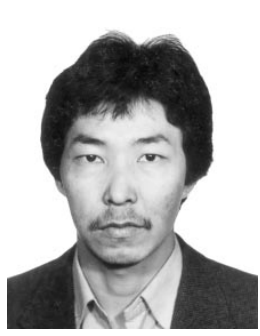

Zhanybai T. Zhusubaliyev was born in Osh Province, Kirghiz, U.S.S.R., in 1958. He received the graduate degree from The Tomsk State University for Control Systems and Electronics (TUSUR), Tomsk, U.S.S.R. in 1982, the Ph.D. degree in electrotechnical complexes and systems from Tomsk State Politechnical University, Tomsk, U.S.S.R., in 1989, and the Dr.Sc. degree in nonlinear dynamics and chaos in relay and pulsewidth modulated control systems from Kursk State Technical University (KSTU), Kursk, Russia, in 2002.

From 1982 to 1992, he was a Researcher in the Research Institute of Automation and Electromechanics (RIAE), Tomsk, U.S.S.R. He is currenlty a Professor in the Department of Computer Science,KSTU. He is a coauthor of more than 80 scientific papers and two books. His research interests include complex dynamics and chaos in piecewise-smooth systems, applications of nonlinear dynamics to power converters, and to relay and pulse control systems.

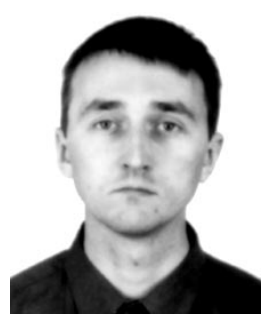

Evgeniy A. Soukhoterin received the M.Sc. degree in computer science from the Kursk State Technical University (KSTU), Kursk, Russia, in 2000. He is currently working toward the doctoral degree at the same university.

$\mathrm{He}$ is also a member of the Scientific Group in Nonlinear Dynamics at KSTU. His research interests include complex dynamics of power electronics devices, quasi-periodicity and resonance phenomena in piecewise-smooth dynamical systems. Mr. Soukhoterin is coauthor of five scientific papers published in leading international journals within the field of nonlinear dynamics.

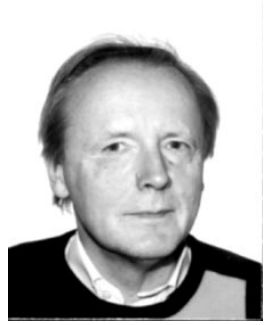

Erik Mosekilde was born in Aarhus, Denmark, in 1941. He received the M.Sc. degree in electrical engineering, the Ph.D. degree in solid-state physics, both from The Technical University of Denmark (DTU), Lyngby, Denmark, in 1966, and 1968, respectively, and the Dr.Sc. degree in semiconductor instabilities from The University of Copenhagen, Copenhagen, Denmark, in 1977.

In 1970, he was a Postdoctoral Fellow at IBM Watson Research Center, Yorktown Heights, New York, and since then, he has been a Visiting Scientist at a number of different universities in Europe and North America. He is a Professor in nonlinear dynamics and Head of the Department of Physics at DTU. His main interests are modeling of complex systems and applications of nonlinear dynamics. He is coauthor of 190 scientific papers and of several books, including two recent books, Topics in Nonlinear Dynamics (Singapore: World Scientific, 1997) and Chaotic Synchronization (Singapore: World Scientific, 2002) 